\title{
Development and applications of a nasopharyngeal carcinoma Tet-Off cell line
}

\author{
YU-PENG FENG ${ }^{1 *}$, QI-CAI LIU ${ }^{4 *}$, JUN-FENG ZHU ${ }^{3}$, FU-KANG XIE ${ }^{1}$ and LI LI $^{2}$ \\ ${ }^{1}$ Department of Histology and Embryology, Zhongshan School of Medicine, Sun Yat-Sen University, Guangzhou 510080; \\ ${ }^{2}$ State Key Laboratory of Oncology in Southern China, Imaging Diagnosis and Interventional Center, \\ Cancer Center; ${ }^{3}$ Department of Pathology, The First Affiliated Hospital, Zhongshan School of Medicine, \\ Sun Yat-Sen University, Guangzhou 510060; ${ }^{4}$ Experimental Medical Research Center, \\ Guangzhou Medical College, Guangzhou 510182, P.R. China
}

Received November 2, 2010; Accepted February 18, 2011

DOI: $10.3892 / \mathrm{ol} .2011 .262$

\begin{abstract}
The conditional activation and inactivation of target gene expression in a nasopharyngeal carcinoma (NPC) cell line is beneficial for the study of the roles of NPC-related genes. Based on the Tet-Off Advanced system, a NPC S18 Tet-Off cell line was developed by stable transfection of a pTetOff Advanced vector (regulator plasmid in Tet-Off Advanced system) into NPC S18 cells. Doxycycline-dependent regulators expressed in the S18 Tet-Off cells were examined by transient and stable transfection of pTRE-Tight-Luc. The S18 Tet-Off-Luc clone selected by stable transfection of pTRETight-Luc into S18 Tet-Off cells expressed firefly luciferase under tight control of doxycycline in a time- and dosedependent manner. To test applications of the S18 Tet-Off cell line in the study of gene function, the impact of ferritin heavy chain (FTH1) gene on NPC cell growth was examined. The S18 Tet-Off-FTH1 clone was developed by stably transfecting pTRE-Tight-FTH1 (response plasmid harboring FTH1) into S18 Tet-Off cells. FTH1 levels in the S18 Tet-Off-FTH1 clone were semi-quantitatively regulated in response to varying concentrations of doxycycline. A cell proliferation assay showed that a high expression of FTH1 (cells grown in the absence of doxycycline) reduced cell growth, while moderate FTH1 overexpression (cells grown in $0.1 \mathrm{ng} / \mathrm{ml}$ doxycycline)
\end{abstract}

Correspondence to: Dr Li Li, Imaging Diagnosis and Interventional Center, Cancer Center, Sun Yat-Sen University, 651 Dongfeng Road East, Guangzhou 510060, P.R. China

E-mail: 1i2@mail.sysu.edu.cn

Dr Fu-Kang Xie, Department of Histology and Embryology, Zhongshan School of Medicine, Sun Yat-Sen University, Guangzhou 510080, P.R. China

E-mail: frankxie2000@yahoo.com

${ }^{*}$ Contributed equally

Key words: doxycycline, nasopharyngeal carcinoma, Tet-Off system, transcription regulation, ferritin heavy chain had no adverse effect on cell growth. In conclusion, the S18 Tet-Off cell line provides a proven genetic background for convenient access to controllable gene expression in NPC.

\section{Introduction}

Nasopharyngeal carcinoma (NPC) is a non-lymphomatous squamous cell carcinoma occurring in the epithelial lining of the nasopharynx. NPC occurs frequently in the populations of Southern China, Northern Africa and Alaska (1). The combination of radiotherapy and chemotherapy is currently the standard treatment strategy. However, the optimal regimens of chemotherapy and radiotherapy have yet to be determined (2). The poor survival rate and high recurrence risk require that novel therapeutic approaches be developed. The therapeutic strategy targeting specific molecules and immunotherapy may improve the of outcome of NPC (3). However, the development of novel strategies is limited due to insufficient understanding of the genes related to NPC development and the lack of conventional cell and animal models for monitoring genes affecting tumor growth and metastasis.

NPC cell line S18, a subclone that was isolated from the human NPC undifferentiated cells (CNE-2 cells) (4), exhibits easy migration and invasion in vitro when compared to CNE-2 cells. Moreover, the S18 xenografted cancer model has a significant metastatic potential and maintains a similar growth rate to the in situ cancer.

Regulation of the expression of target genes in the S18 cell line, is considered to be of great significance for the investigation of target genes in NPC. The Tet-Off Advanced System is a well-developed gene regulation tool in eucaryon $(5,6)$. The Tet-Off Advanced system comprises two key components: the doxycycline-dependent regulator, such as pTet-Off Advanced, and the response element containing the target gene, such as pTRE-Tight-X. The Tet-Off Advanced system aims to create a double-stable cell line that contains integrated copies of the regulator and response plasmids. In this cell line, transcription of the gene of interest is maintained in the 'off' state by the presence of doxycycline in the culture medium, while transcription induction may occur following the removal of doxycycline. Although this system appears to 
be beneficial, more studies are required to create an inducible system. Moreover, the efficacy of doxycycline-controlled gene induction is known to be affected by cell types (7-9). To the best of our knowledge, no study has reported whether this system can confirm a high efficacy of transcriptional induction of target genes in NPC cell lines. In the present study, a NPC S18 Tet-Off cell line was developed, which effectively expressed a doxycycline-dependent regulator, and had potent transcriptional activity of genes of interest with a low background.

\section{Materials and methods}

Cell culture. Human NPC S18 cells (kindly provided by Dr Chaonan Qian) were grown in high glucose (Gibco) Dulbecco's modified Eagle's minimal essential medium (DMEM) containing 10\% Tet system-approved fetal bovine serum (FBS; Clontech). S18 Tet-Off cells were grown in complete DMEM medium containing $100 \mu \mathrm{g} / \mathrm{ml}$ G418 (Clontech). The S18 Tet-Off-Luc and Tet-Off-FTH1 clones were grown in complete DMEM containing $100 \mu \mathrm{g} / \mathrm{ml} \mathrm{G} 418$ and $100 \mu \mathrm{g} / \mathrm{ml}$ hygromycin (Alexis) with or without $10 \mathrm{ng} / \mathrm{ml}$ doxycycline (Alexis). For inducible experiments, cells maintained in the 'off' state of gene expression by $10 \mathrm{ng} / \mathrm{ml}$ doxycycline were passaged. Briefly, the cells were washed twice with phosphate-buffered solution (PBS) prior to trypsinization. Following trypsinization and harvesting, the cells were washed twice with PBS, and grown in fresh medium with or without various concentrations of doxycycline.

Transfection protocol. Cells were transfected using Lipofectamine 2000 (Invitrogen) according to modified manufacturer's instructions. For transient transfection, cells $\left(2 \times 10^{4}\right)$ were plated in $100 \mu 1$ of complete cell growth medium on a 96-well plate and incubated for $24 \mathrm{~h}$ until a cell confluence of $50-60 \%$ was achieved. DNA-Lipofectamine 2000 was prepared by combining the diluted plasmid DNA [0.22 $\mathrm{mg}$ in $25 \mathrm{ml}$ of Opti-MEM (Invitrogen)] with Lipofectamine 2000 $(0.15 \mathrm{ml}$ in $25 \mathrm{ml}$ of Opti-MEM) followed by incubation at room temperature for $20 \mathrm{~min}$. The DNA-Lipofectamine 2000 reagent complex was then directly added to each well, followed by mixing and subsequent incubation at $37^{\circ} \mathrm{C}$. After $6 \mathrm{~h}$, the medium was replaced with fresh complete cell growth medium with or without $10 \mathrm{ng} / \mathrm{ml}$ doxycycline followed by incubation for $48 \mathrm{~h}$. Firefly luciferase activity was then determined.

For stable transfection, $5 \times 10^{5}$ cells in $500 \mu 1$ of complete cell growth medium were seeded on a 24-well plate. After 16-24 h of culture, the cells were transfected with DNA-Lipofectamine 2000 reagent complex $(0.8$ or $0.84 \mu \mathrm{g}$ of plasmid in $50 \mu 1$ of Opti-MEM mixed with $0.5 \mu 1$ of Lipofectamine 2000 in $50 \mu$ l of Opti-MEM) when cell confluence of approximately $90 \%$ was achieved. All the plasmids were digested with restriction enzyme ScaI. One day posttransfection, the cells were washed, trypsinized and divided equally onto two $10-\mathrm{cm}$ plates. Following $24 \mathrm{~h}$ of incubation, either $500 \mu \mathrm{g} / \mathrm{ml} \mathrm{G} 418$ or G418 plus $250 \mu \mathrm{g} / \mathrm{ml}$ hygromycin was added to the medium. After 16 days, antibiotic-resistance clones were selected, using cloning rings, and analyzed. Under these conditions, the transfection efficacy was $>80 \%$, with low cytotoxicity detected $48 \mathrm{~h}$ after transfection with pEGFP-C1 under identical transfection conditions.

Firefly luciferase activity assay. Cells were washed twice with PBS, lysed and then assayed for firefly luciferase activity with the dual-luciferase reporter assay (for the transient transfection that requires Renilla luciferase to normalize the transfection efficacy) or the luciferase assay system, according to the manufacturer's instructions (Promega, Madison, WI, USA). The firefly luciferase activity in each cell lysate was measured with the GloMax 96 Microplate Luminometer (Promega) according to the standard protocol, and the protein concentration was determined using the BCA method (Pierce, Rockford, IL, USA). Luminescence data were expressed as relative light units (measured in $10 \mathrm{~s}$ ) per milligram of protein.

Construction of plasmids and reverse transcription-PCR $(R T-P C R)$. The human ferritin heavy chain gene (FTH1) with Kozak sequence was amplified by RT-PCR from the total RNA of S18 cells. FTH1 gene was then subcloned into the SmaI site of pUC119 yielding pUC119-FTH1. Following sequencing, the fragment from pUC119-FTH1 after digestion by EcoRI and $K p n I$ was cloned into the same site of pTRE-Tight yielding pTRE-Tight-FTH1. For RT-PCR, the first strand cDNA was reverse transcribed with oligo (dT) primer. The full-length FTH1 with Kozak sequence was amplified using PrimeSTAR HS DNA Polymerase (Takara). The primers were forward, 5'-GAATTCGCCACCATGACGACCGCGTCCACCTC-3' and reverse, 5'-AGATCTGGTACCTTTAGCTTTCATTATCA CTGTC-3'.

Western blot analysis. Cells were lysed in RIPA buffer $[50 \mathrm{mM}$ Tris $\mathrm{pH} 8.0,150 \mathrm{mM}$ sodium chloride, $1.0 \%$ Triton X-100 (v/v), $0.5 \%$ sodium deoxycholate and $0.1 \% \mathrm{SDS}(\mathrm{w} / \mathrm{v})]$, agitated for $30 \mathrm{~min}$ at $4^{\circ} \mathrm{C}$ and centrifuged at $12,000 \mathrm{xg}$ for $15 \mathrm{~min}$. The concentration of total proteins was determined using BCA. Total proteins $(25 \mu \mathrm{g})$ in equal volume of $2 \mathrm{X}$ Laemmli buffer were then denatured and subjected to 12\% SDS-PAGE. The proteins were transferred onto acetyl cellulose membranes which were subsequently blocked in 5\% non-fat milk in TBST (20 mM Tris pH 7.6, $137 \mathrm{mM}$ NaCL, 0.1\% Tween-20). The membranes were incubated with primary antibodies overnight [rabbit anti-FTH1 1:1,000 (Santa Cruz Biotechnologies, Santa Cruz, CA, USA) and rabbit anti-GAPDH 1:2,000 (GenScript, Piscataway, NJ, USA)]. Following washing, the membranes were treated with secondary antibody [HRP-conjugated goat anti-rabbit 1:5,000 (Invitrogen)] and visualized by enhanced chemiluminescence.

MTT assay. Cell proliferation was measured by 3-(4,5-dimethylthiazol-2-yl)-2,5-diphenyltetrazolium bromide (MTT; Sigma) assay. Cells were plated onto 96-well plates and maintained in $100 \mu \mathrm{l}$ of DMEM (supplemented with different concentrations of doxycycline) for 3 days. The experiment was repeated three times for each group. After 24, 48 and $72 \mathrm{~h}$ of incubation, $20 \mu \mathrm{l}$ MTT was added to each well and the incubation was maintained for an additional $4 \mathrm{~h}$ at $37^{\circ} \mathrm{C}$. At the end of the incubation period, $150 \mu \mathrm{l}$ DMSO was added to each well and throroughly mixed for $10 \mathrm{~min}$. Absorbance at $570 \mathrm{~nm}$ was then determined. 
Table I. Analysis of firefly luciferase activity induction in the S18 Tet-Off clones.

\begin{tabular}{lccc}
\hline Cell clone & \multicolumn{2}{c}{ Firefly luciferase activity (RLU/ $\mu$ g protein) } & Regulation factor \\
\cline { 2 - 3 } & Dox $^{+}$ & Dox $^{-}$ & 274 \\
A5 & $175 \pm 13$ & $48,062 \pm 4,052$ & 236 \\
B18 & $397 \pm 18$ & $93,619 \pm 8,466$ & 190 \\
B7 & $193 \pm 16$ & $36,835 \pm 1,613$ & 146 \\
B4 & $120 \pm 11$ & $17,623 \pm 1,489$ & 131 \\
A12 & $75 \pm 10$ & $9,836 \pm 801$ & 130 \\
B15 & $228 \pm 12$ & $29,571 \pm 2,763$ & 117 \\
A21 & $201 \pm 12$ & $23,523 \pm 1,045$ & 42 \\
A9 & $74 \pm 5$ & $3,115 \pm 188$ & 28 \\
B23 & $96 \pm 9$ & $2,653 \pm 267$ & 23 \\
B19 & $77 \pm 6$ & $1,766 \pm 189$ & \\
\hline
\end{tabular}

S18 cells were stably transfected with the pTet-Off Advanced plasmid, and clones resistant to G418 were selected and transiently transfected with pTRE-Tight-Luc. Cell culture was performed in the presence $\left(\right.$ Dox $\left.^{+}\right)$or absence $\left(\right.$Dox $\left.^{-}\right)$of $10 \mathrm{ng} / \mathrm{ml}$ doxycycline for $48 \mathrm{~h}$, followed by harvesting and measurement of firefly luciferase activity. Data were normalized by the Renilla luciferase activity (introduced by cotransfection with pRL-TK to normalize the transfection efficacy). Of the 52 G418-resistant clones, 10 exhibited a $>20$-fold regulation factor. Data from four independent transfection experiments are expressed as the means \pm SD.

Table II. Analysis of firefly luciferase activity induction in the S18 Tet-Off-Luc clones.

\begin{tabular}{|c|c|c|c|}
\hline \multirow[t]{2}{*}{ Cell clone } & \multicolumn{2}{|c|}{ Firefly luciferase activity (RLU/ $\mu$ g protein) } & \multirow[t]{2}{*}{ Regulation factor } \\
\hline & $\operatorname{Dox}^{+}$ & $\operatorname{Dox}^{-}$ & \\
\hline D7 & $0.89 \pm 0.17$ & $3,281 \pm 352$ & 3,671 \\
\hline D25 & $2.47 \pm 0.33$ & $5,280 \pm 188$ & 2,138 \\
\hline D18 & $29.3 \pm 2.60$ & $44,491 \pm 1,189$ & 1,534 \\
\hline D3 & $0.96 \pm 0.09$ & $1,177 \pm 167$ & 1,225 \\
\hline
\end{tabular}

The S18 Tet-Off cell line was co-transfected with pTRE-Tight-Luc and the linear hygromycin marker. Clones resistant to G418 and hygromycin were selected and each clone was grown in the presence or absence of $10 \mathrm{ng} / \mathrm{ml}$ doxycycline for $48 \mathrm{~h}$. The firefly luciferase activity was then assayed. Of the 24 clones, 4 exhibited a $>1,000$-fold induction. The clone with the lowest background level (D7) was termed the S18 Tet-Off-Luc cell line and subcloned for further experiments. Data from four independent experiments were expressed as the means \pm SD.

\section{Results}

Generation of S18 Tet-Off clones. NPC S18 cells were transfected with the pTet-Off Advanced plasmid, the G418-resistant clones were isolated and the inducible firefly luciferase activity was detected following transient transfection of pTRE-TightLuc into each clone. Among the 52 clones identified, the firefly luciferase expression in 10 clones during induction was 20 -fold higher than that at the non-induced state (Table I), indicating the 10 clones that expressed the functional regulator protein. Of the 10 clones, the cell clone (A9) with a 42-fold increase in the firefly luciferase expression was selected as the host cell line for the development of double-stable cells (S18 Tet-Off cell line).

Generation of clones with inducible firefly luciferase activity. To investigate whether the S18 Tet-Off cell line maintains a high induction activity of firefly luciferase and a low basal expression, clones were prepared by stable transfection with pTRE-Tight-Luc into the cell line. Of the 24 clones, 4 exhibited a $>1,000$-fold increase in induction (Table II). These results suggest that it is relatively simple to develop stable clones that show at least 1,000-fold inducible transcription activity based on the host cell line. The D7 clone with the lowest basal expression and the highest induction activity of firefly luciferase was termed the S18 Tet-Off-Luc cell line and subcloned for further experiments.

Time kinetics of inducible firefly luciferase expression. The time course of firefly luciferase activity of the S18 Tet-Off-Luc cell line was analyzed in the absence or presence of doxycycline. As shown in Fig. 1A, the depletion of doxycycline led to a rapid induction of firefly luciferase activity. Luciferase activity increased by 775-fold within $3 \mathrm{~h}$ and by 3,321-fold in $12 \mathrm{~h}$; the peak level was reached within $24 \mathrm{~h}$. To investigate whether residual doxycycline in culture medium inhibits the 
A

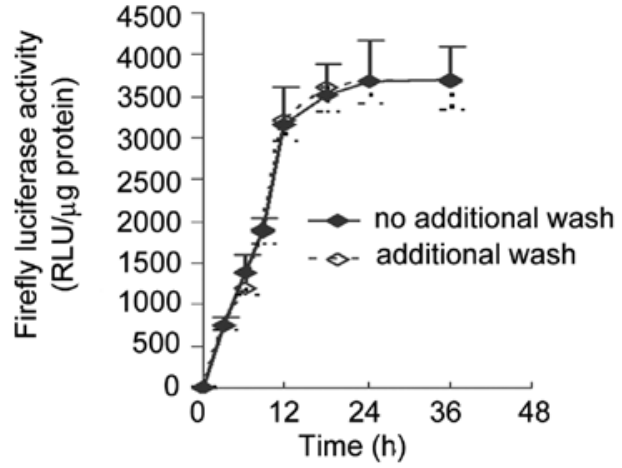

B

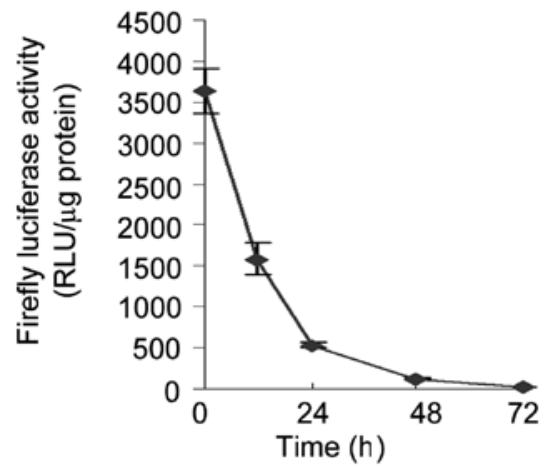

C

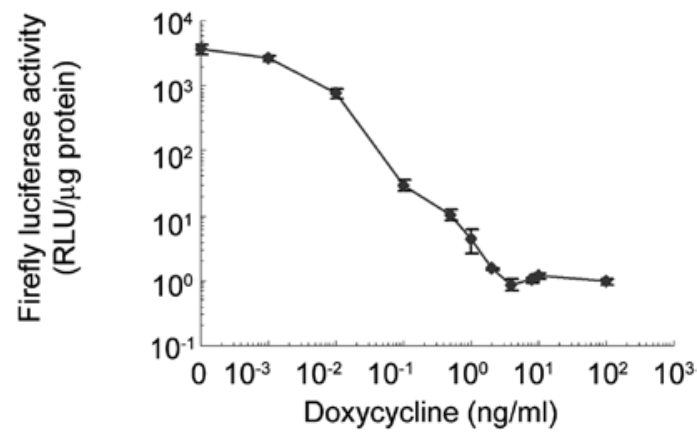

Figure 1. Time- and dose-dependent firefly luciferase activity of the S18 TetOff-Luc cell line. (A) Cells were grown in the medium containing $10 \mathrm{ng} / \mathrm{m}$ doxycycline for at least 5 days. The cells were then transferred into doxycyclinefree medium with or without an additional wash after $3 \mathrm{~h}$ of the initial remova of doxycycline. The cell extracts were harvested at the designated time points and examined for luciferase activity. The two time courses almost overlapped each other, leading to a rapid induction of firefly luciferase activity with or without an additional wash following the depletion of doxycycline, indicating that residual doxycycline did not inhibit maximum transcription activity. (B) Cells were grown in the medium without doxycycline for 5 days. The cells were then transferred to a medium containing $10 \mathrm{ng} / \mathrm{ml}$ doxycycline. The cells were harvested at the designated time points and examined for luciferase activity. A rapid reduction of firefly luciferase activity was observed in the presence of doxycycline. (C) Cells were grown in the medium containing $10 \mathrm{ng} / \mathrm{ml}$ doxcycline for 5 days and then in fresh medium with various concentrations of doxycycline. Incubation was performed for $48 \mathrm{~h}$ and cells were harvested and examined for firefly luciferase activity. The induction of luciferase activity by doxycycline in S18 Tet-Off-Luc cells occurred in a dose-dependent manner. The doxycycline concentration ranged between 0 and $4 \mathrm{ng} / \mathrm{ml}$, within which the expression of firefly luciferase was the most sensitive to doxycycline induction. Data from three independent experiments are expressed as the means $\pm \mathrm{SD}$.

full gene expression (maximum transcription activity), the cells were washed once with PBS $3 \mathrm{~h}$ after initial removal of doxycycline, and the luciferase activity was analyzed. As shown in Fig. 1A, the two time courses almost overlapped. As shown in Fig. 1B, a similar rapid reduction of firefly luciferase
A

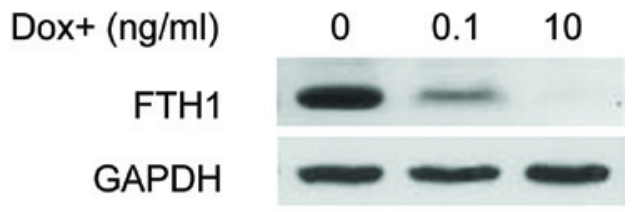

B

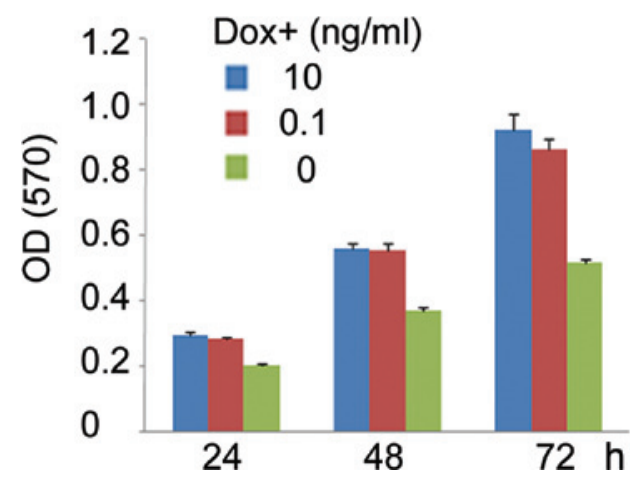

Figure 2. FTH1 gene levels in the S18 Tet-Off-FTH1 clone regulated by various concentrations of doxycycline and its effect on cell growth. (A) FTH1 levels induced by different concentrations of doxycycline were detected by Western blotting. In the S18 Tet-Off-FTH1 clone, the expression of FTH1 was semi-quantitatively controlled by doxycycline. (B) Effect of various levels of FTH1 on the growth of cells. A high expression of FTH1 at $0 \mathrm{ng} / \mathrm{ml}$ doxycycline reduced cell growth, while no obvious growth changes were observed by a slight overexpression of FTH1 $(0.1 \mathrm{ng} / \mathrm{ml})$. The growth rate of cells at 10 $\mathrm{ng} / \mathrm{ml}$ doxycycline was used as the control. Values given are the means $\pm \mathrm{SD}$ $(\mathrm{n}=3 ; \mathrm{P}<0.05$, two-tailed unpaired t-test).

activity was observed in the presence of doxycycline. The luciferase activity dropped to $43.7 \%$ of its initial level within $12 \mathrm{~h}$ and to $<15 \%$ in $24 \mathrm{~h}$.

Dose-dependent expression of firefly luciferase by doxycycline. S18 Tet-Off-Luc cells were grown in medium containing 10 $\mathrm{ng} / \mathrm{ml}$ doxycycline for 5 days and then in medium containing various concentrations of doxycycline for $48 \mathrm{~h}$. The cell extracts were then analyzed for firefly luciferase activity. As shown in Fig. 1C, the firefly luciferase activity was completely inhibited at $4 \mathrm{ng} / \mathrm{ml}$ doxycycline, an event that was also observed at 100 $\mathrm{ng} / \mathrm{ml}$. A stepwise reduction in the concentration of doxycycline gradually increases the activity of firefly luciferase from complete inhibition to an increase of 3,600-fold. This result showed that induction of the luciferase expression by doxycycline in S18 Tet-Off-Luc cells occurred in a dose-dependent manner. The doxycycline concentration ranged between 0 and $4 \mathrm{ng} / \mathrm{ml}$, within which the expression of firefly luciferase was the most sensitive to doxycycline induction.

Expression of human ferritin heavy chain controlled by doxycycline. For further applications of the S18 Tet-Off cell line, the clone inducibly expressing FTH1 was developed. The construct of pTRE-Tight-FTH1 was stably transfected into S18 Tet-Off cells. FTH1 levels in clones were determined by Western blotting. One of these clones expressing the highest levels of FTH1 in the absence of doxycycline with a low background level in the presence of $10 \mathrm{ng} / \mathrm{ml}$ doxycycline was selected and termed S18 Tet-Off-FTH1 clone. By adjusting the concentrations of doxycycline, the semi-quantitative expression of FTH1 was achieved in the S18 Tet-Off-FTH1 
clone. Fig. 2A shows that in the S18-Off-FTH1 cell line, FTH1 expression was maximally inhibited at a concentration of $10 \mathrm{ng} / \mathrm{ml}$ doxycycline and increased to a moderate level at $0.1 \mathrm{ng} / \mathrm{ml}$, and then completely depressed when doxycycline was removed.

Effect of human ferritin heavy chain overexpression on NPC S18 cell proliferation. The S18-Off-FTH1 clone is maintained in culture in the absence of doxycycline for up to 2 months without evident signs of toxicity. However, it was observed that the clone achieved confluence more rapidly in the presence of doxycycline than in the absence thereof. Doxycycline at $10 \mathrm{ng} /$ $\mathrm{ml}$ had no evident effects on cell growth. To assess the impact of FTH1 on cell proliferation, a MTT assay that measures mitochondrial activity was used (Fig. 2B). The results showed a correlation between the effect on cell viability and the FTH1 levels. When cells were grown in the absence of doxycycline, a significantly high overexpression of FTH1 caused at least a $30 \%$ decrease in cell growth rate at $48 \mathrm{~h}$. When cells were grown at $0.1 \mathrm{ng} / \mathrm{ml}$ doxycycline, the moderate overexpression of FTH1 did not affect cell growth.

\section{Discussion}

Among head and neck cancers, NPC is highly susceptible to lymph node metastasis. Undifferentiated NPCs have a higher rate of local control after treatment, but a higher incidence of distant metastasis than differentiated NPCs $(10,11)$. The undifferentiated NPC S18 cell line, not only showed characteristics of advanced cancers, but also had a high potential of lymph node metastasis. Therefore, S18 cells serve as an option for the development of a cell model in which the S18 target gene is conditionally overexpressed.

In this study, following the stable transfection of pTet-Off Advanced plasmid into S18 cells, G418-resistant clones were selected and analyzed using the transient luciferase expression assay. In the investigation of 10 clones with an increase of luciferase activity of more than 20-fold, the expression levels in the absence of doxycycline varied by 53 -fold, whereas the expression levels at $10 \mathrm{ng} / \mathrm{ml}$ doxycycline varied by less than 6-fold.

This clonal variation is most likely contributed to the variation in the concentration of regulator proteins in each clone (12). According to the manufacturer's instructions of the Tet-Off Advanced system (Clontech), only clones exhibiting 20- to 50-fold induction were selected to develop double-stable cell lines. We selected the clone with a 42-fold induction as the host cell line. Following stable transfection with pTRE-TightLuc into this clone, clones with more than 1,000-fold induction of transcriptional activity were developed. The basal expression level (RLU=0.89, $\mathrm{n}=4, \mathrm{SD}=0.17$ ) of the double-stable cell line (S18 Tet-Off-Luc) in the presence of $4 \mathrm{ng} / \mathrm{ml}$ doxycycline was comparable to that of the background level. This extremely low background level is similar to that of well-established double-stable Tet controlled cell lines, such as HeLa (clone X1) and CHO AA8 (clone 19) $(5,12)$. As shown in the majority of studies, if a low level of background expression is achieved, a regulation factor of between 50- and approximately 1,000-fold is sufficient (13). Therefore, the 42-fold clone was termed S18 Tet-Off cell line.
Rennel and Gerwins previously reported that residual doxycycline binding to cells or extracellular matrix prevents full gene induction in the Tet-Off system. Moreover, a robust and rapid transgene expression is induced in certain cell lines if doxycycline is removed by washing $3 \mathrm{~h}$ after the initial removal of doxycycline (14). We found that the additional wash was not required for the double-stable cell lines based on the S18 Tet-Off cell line, making the operation more convenient.

As shown in Fig. 1C, the transcription activity was quantitatively regulated by doxycycline. The doxycycline at between 0 and $4 \mathrm{ng} / \mathrm{ml}$ allowed adjustment of promoter activity within a range of three orders of magnitude. This may allow assessment of quantitative parameters of gene function.

To further test the application of the S18 Tet-Off cell line, another clone that inducibly expressed candidate MRI reporter gene FTH1 $(15,16)$ was successfully created. As an MRI candidate reporter, evidence of its safety warrants further investigation of its practical applications. Thus, in the present study, the impact of FTH1 overexpression on NPC S18 cell growth was addressed. The highest expression of FTH1 (grown in the absence of doxycycline) reduced cell growth. However, a moderate overexpression of FTH1 (grown in $0.1 \mathrm{ng} / \mathrm{ml}$ doxycycline) in NPC S18 cells did not reduce cell growth. These results were in accordance with those of Cozzi et al (18). Findings of that study showed that the overexpression of FTH1 in HeLa cells induced an iron-deficient phenotype with significantly reduced cell growth. In a previous study, overexpression of FTH1 in C6 glioma cell lines (19), A549 cells (16) and stem cells (20) did not reduce cell growth. However, Cheng et al (21) found that a significant reduction in growth rate was observed in C6 glioma cell lines under markedly high FTH1 transgene expression ( $>500 \%$ of the level in mouse embryonic stem cells described in their study). On the other hand, in other S18 double-stable clones, those with low FTH1 levels did not reduce cell growth (data not shown). It was hypothesized that a significantly high expression of FTH1 decreases cell growth in certain types of cells, whereas a moderate level of FTH1 expression may be safe.

To investigate the role of genes relative to NPC, a NPC cell line stably expressing the genes is a powerful tool. Routinely, target genes under the general and non-controlled promoter, including CMV promoter, are transfected into host cells, and then clones with high expression levels are selected to investigate the involvement of these clones in NPC. However, this process leads to certain issues: i) To develop stable clones with a high expression of level of target genes is impossible if the target genes are toxic to cells. ii) If the gene of interest has no evident cell cytotoxity, a high expression of target genes continues for the whole clone-selected process. Chronic alterations may exist, however, and these changes are often not easy to detect. For example, a high expression of FTH1 reduces NPC S18 cell growth, and does not form clones in the early clone-selected days. Clones with low expression levels are usually selected. If this selection occurs, it is difficult to judge the impact of FTH1 on cell growth. iii) The expression levels vary largely as a result of different vector integration sites. In order to study the biological properties on different levels of the target gene, more cell clones should be selected and investigated. However, this process is time-consuming 
and not cost-effective. A gene-controllable system would be beneficial. Therefore, the NPC S18 Tet-Off cell line is an effective tool that provides a proven genetic background for gene regulation. Using the NPC S18 Tet-Off cell line, only one-step transfection is required to produce a cell model in which the target genes can be specifically and tightly regulated. The characteristically low level of basal activity is beneficial for studies on novel genes that may disrupt the cell cycle, induce apoptosis or exhibit cytotoxicity, in the case that experiments depend on the absolute control of the background of the gene expression level.

\section{Acknowledgements}

This study was supported by the National Natural Science Foundation of China (No. 80171207 and 39970237), the Fundamental Research Funds for the Central Universities (No. 10ykjcll), and the Open Funds of State Key Laboratory of Oncology in Southern China.

\section{References}

1. Wei WI and Sham JS: Nasopharyngeal carcinoma. Lancet 365: 2041-2054, 2005.

2. Lu H, Peng LX, Yuan XB, et al: Concurrent chemoradiotherapy in locally advanced nasopharyngeal carcinoma: a treatment paradigm also applicable to patients in Southeast Asia. Cancer Treat Rev 35: 345-353, 2009 .

3. Agulnik M and Epstein JB: Nasopharyngeal carcinoma: current management, future directions and dental implications. Oral Oncol 44: 617-627, 2008.

4. Qian CN, Berghuis B, Tsarfaty G, et al: Preparing the 'soil': the primary tumor induces vasculature reorganization in the sentine lymph node before the arrival of metastatic cancer cells. Cancer Res 66: 10365-10376, 2006.

5. Gossen $M$ and Bujard H: Tight control of gene expression in mammalian cells by tetracycline-responsive promoters. Proc Natl Acad Sci USA 89: 5547-5551, 1992.

6. Urlinger S, Baron U,Thellmann M, Hasan MT,Bujard H and Hillen $\mathrm{W}$ : Exploring the sequence space for tetracycline-dependent transcriptional activators: novel mutations yield expanded range and sensitivity. Proc Natl Acad Sci USA 97: 7963-7968, 2000.

7. Gossen M and Bujard H: Efficacy of tetracycline-controlled gene expression is influenced by cell type: commentary. Biotechniques 19: 213-217, 1995.
8. Ackland-Berglund CE and Leib DA: Efficacy of tetracyclinecontrolled gene expression is influenced by cell type. Biotechniques 18: 196-200, 1995.

9. Howe JR, Skryabin BV, Belcher SM, Zerillo CA and Schmauss C: The responsiveness of a tetracycline-sensitive expression system differs in different cell lines. J Biol Chem 270: 14168-14174, 1995.

10. Marks JE, Phillips JL and Menck HR: The National Cancer Data Base report on the relationship of race and national origin to the histology of nasopharyngeal carcinoma. Cancer 83: 582-588, 1998.

11. Reddy SP, Raslan WF, Gooneratne S, Kathuria S and Marks JE: Prognostic significance of keratinization in nasopharyngeal carcinoma. Am J Otolaryngol 16: 103-108, 1995.

12. Wang XS, Hu CS, Ying HM, Zhou ZR, Ding JH and Feng Y: Patterns of retropharyngeal node metastasis in nasopharyngeal carcinoma. Int J Radiat Oncol Biol Phys 73: 194-201, 2009.

13. Freundlieb S, Schirra-Muller C and Bujard H: A tetracycline controlled activation/repression system with increased potential for gene transfer into mammalian cells. J Gene Med 1: 4-12, 1999.

14. Rennel E and Gerwins P: How to make tetracycline-regulated transgene expression go on and off. Anal Biochem 309: 79-84, 2002.

15. Cohen B, Ziv K, Plaks V, et al: MRI detection of transcriptional regulation of gene expression in transgenic mice. Nat Med 13: 498-503, 2007.

16. Genove G, DeMarco U, Xu H, Goins WF and Ahrens ET: A new transgene reporter for in vivo magnetic resonance imaging. Nat Med 11: 450-454, 2005.

17. Zurkiya O, Chan AWS and Hu XP: MagA is sufficient for producing magnetic nanoparticles in mammalian cells, making it an MRI reporter. Magn Reson Med 59: 1225-1231, 2008.

18. Cozzi A, Corsi B, Levi S, Santambrogio P, Albertini A and Arosio P: Overexpression of wild type and mutated human ferritin $\mathrm{H}$-chain in HeLa cells: in vivo role of ferritin ferroxidase activity. J Biol Chem 275: 25122-25129, 2000.

19. Cohen B, Dafni H, Meir G, Harmelin A and Neeman M: Ferritin as an endogenous MRI reporter for noninvasive imaging of gene expression in C6 glioma tumors. Neoplasia 7: 109-117, 2005.

20. Naumova AV, Reinecke H, Yarnykh V, Deem J, Yuan C and Murry CE: Ferritin overexpression for noninvasive magnetic resonance imaging-based tracking of stem cells transplanted into the heart. Mol Imaging 9: 201-210, 2010.

21. Liu J, Cheng ECH, Long RC, et al: Noninvasive monitoring of embryonic stem cells in vivo with MRI transgene reporter. Tissue Engineering Part C Methods 15: 739-747, 2009. 